Diabetologe 2012 $\cdot 8: 203-204$

DOI 10.1007/s11428-012-0880-7

Online publiziert: 6 . April 2012

(c) Springer-Verlag 2012
W.A. Scherbaum ${ }^{1} \cdot$ R. Landgraf ${ }^{2}$

${ }^{1}$ Klinik für Endokrinologie, Diabetologie und Rheumatologie,

European Training Centre in Endocrinology and Metabolism, Universitätsklinikum Düsseldorf

${ }^{2}$ Deutsche Diabetes-Stiftung, München

\title{
Implementierung von Diabetesleitlinien
}

\section{Wichtige Rolle der medizinischen Pflegekräfte}

Bei der Entwicklung evidenzbasierter Behandlungsleitlinien hat die Deutsche Diabetes-Gesellschaft (DDG) eine Vorreiterrolle eingenommen [1]. Inzwischen sind hochwertige Leitlinien auf der Basis großer klinischer Studien für fast alle medizinischen Fachgebiete erstellt worden. Die Diabetesleitlinien DDG wurden von Anfang an mit anderen Fachgebieten abgestimmt. Diese Aufgabe wurde inzwischen an das Ärztliche Zentrum für Qualität in der Medizin (ÄZQ) übertragen, und dieses hat die Koordination einer Reihe evidenzbasierter Nationaler Versorgungsleitlinien (NVL; www.aezq.de) übernommen. In enger Kooperation mit Experten aus den in der Arbeitsgemeinschaft Wissenschaftlich-Medizinischer Fachgesellschaften (AWMF) organisierten Fachgesellschaften, zusammen mit Vertretern aus dem Verband der Diabetesberatungs- und Schulungsberufe in Deutschland (VDBD) sowie Patientenvertretern wurden inzwischen wichtige NVL publiziert: Nierenerkrankungen bei Diabetes, Netzhautkomplikationen, Fußkomplikationen und Neuropathie bei Diabetes. Wichtige weitere NVL wie Schulung und Schulungsprogramme sowie Therapieplanung bei Typ-2-Diabetes sind in Bearbeitung. Bei der Bearbeitung dieser Leitlinienserie waren leider keine Vertreter der Pflege eingebunden. Dabei sind nach Ansicht der Autoren die Pflegeberufe von entscheidender Bedeutung bei der Implementierung der Leitlinien.

Bei der praktischen Umsetzung von Leitlinien (systematisch entwickelte
Handlungsempfehlungen für Ärzte) geht es insbesondere darum, die allgemeinen Empfehlungen auf den Einzelfall anzuwenden. Also weg vom „disease management" hin zum „case management“. Dies gilt insbesondere für die hoch komplexen multimorbiden Menschen mit Diabetes. Gary et al. [2] konnten in einer großen Studie bei Amerikanern, die in einer ländlichen Region leben, zeigen, dass der Typ-2-Diabetes besser einzustellen ist, wenn man Krankenschwestern und ein Team von kommunalen nichtärztlichen Kräften des Gesundheitsamts als Case manager einsetzt. Diese Kräfte kontrollierten die Umsetzung der Behandlungsvorgaben und führten $u$. a. auch Hausbesuche durch. Patienten in der durch Personen des kommunalen Gesundheitsamts betreuten Gruppe mussten weniger häufig stationär eingewiesen werden.

Welch et al. [3] publizierten eine Metaanalyse, die sich mit dem Einfluss des Case management durch Pflegekräfte bei Diabetesinterventionsstudien beschäftigten und die Blutglukosekontrolle zum Ziel hatte. Erwartungsgemäß zeigte sich, dass die Einbindung von Pflegekräften in das Case management in hohem Maß effektiv ist. Auf dem Boden der Analyse von 29 Studien wurde gefolgert, dass ein von Pflegekräften geleitetes Case management insbesondere bei Patienten mit schlecht eingestelltem Diabetes die optimale Behandlungsstrategie darstellt. Ishani et al. [4] belegten, dass versierte Pflegekräfte auch für komplexere Aufgaben bei der Behandlung von Menschen mit Dia- betes eingesetzt werden können, wenn sie z. B. das Erreichen der Zielwerte für Blutglukose $/ \mathrm{HbA}_{1 c}$, Blutdruck und „Low-density-lipoprotein“(LDL)-Cholesterin überwachen und anhand von vorgeplanten Algorithmen nachsteuern. Je häufiger die Kontakte mit der Pflegekraft angesetzt waren, umso besser waren die Ergebnisse. Dabei führten die „diabetes nurses“ - in Deutschland wären dies z. B. Diabetesberater/innen - auch Beratungen und praktische Anleitungen zu Lebensstiländerungen durch. Während mit der Einbindung der Pflegekräfte nach einem Jahr 22\% der Patienten die Zielwerte erreicht hatten, waren das bei Standardbehandlung nur 10\%. Diese positiven Ergebnisse wurden von Appel [5] vom Capstone College of Nursing (University of Alabama, AL, USA) so interpretiert, dass aufgrund dieser Daten den Case managers mehr Kompetenzen bei der Betreuung und Behandlung von Menschen mit Diabetes übergeben werden sollten. Dies spielt z. B. bei der Funktion der Diabetesberater/innen und anderen nichtärztlichen medizinischen Fachkräften im Rahmen der integrierten Versorgung beim Diabetes [6] sowie bei der Behandlung geriatrischer Patienten mit Diabetes [7] eine große Rolle und ist auch in einem hohen Maße kostengünstig sowie ressourcensparend [8].

Dem hat die Gesundheitspolitik in der „Richtlinie des Gemeinsamen Bundesausschusses über die Festlegung ärztlicher Tätigkeiten zur Übertragung auf Berufsangehörige der Alten- und Krankenpflege zur selbstständigen Ausübung von 
Heilkunde im Rahmen von Modellvorhaben nach $\$ 63$ Abs. 3c SGB V“ in der Fassung vom 20.10.2011 Rechnung getragen (vom Bundesministerium für Gesundheit noch nicht freigegeben). Danach kann bei „den im Folgenden aufgeführten ärztlichen Tätigkeiten im Rahmen von Modellvorhaben eine Übertragung auf Berufsangehörige der Kranken- und Altenpflege zur selbstständigen Ausübung von Heilkunde erfolgen. Die selbstständige Ausübung von Heilkunde setzt voraus, dass die jeweils erforderliche Qualifikation gemäß $\$ 4$ Abs. 7 des Krankenpflegegesetzes (KrPflG) bzw. $\$ 4$ Abs. 7 des Altenpflegegesetzes (AltPflG) erworben wurde“. Dazu sollen Krankheiten zählen wie:

- Typ-1- und Typ-2-Diabetes,

- chronische Wunden (z. B. Ulcus cruris),

- Hypertonus (ohne Schwangerschaft) und

- Demenz.

Diese Richtlinie birgt jedoch die große Gefahr, dass die jahrelangen Bemühungen einer integrierten Versorgung von Menschen mit Diabetes (Teambetreuung) konterkariert werden.

Was ist der Grund für die Nichteinbindung der Pflege und für die mangelnde Abstimmung mit den Ärzten und Wissenschaftlern? Nach Meinung der Autoren ist dies in dem historischen Umstand begründet, dass bisher für die Pflege in Deutschland keine entsprechende Ausbildung verfügbar war, die den Zugang zur akademischen Medizin erlaubte. Dieses Manko kann nun durch den Studiengang „Master of Science“ der Pflegewissenschaften angegangen werden, den die Studenten mit einer Master-Arbeit als eine Art von Diplom abschließen. Kurzversionen von drei dieser Arbeiten finden Sie exemplarisch in diesem Heft. Dabei geht es im Wesentlichen um Fragen, die vorrangig Pflegekräfte betreffen und die auf den direkten Umgang mit den Patienten sowie die Umsetzung von Therapiemaßnahmen zielen.

Es geht hier also um den Aufbau von Versorgungsforschung, einem Stiefkind in der Forschungslandschaft in Deutschland. Selbstverständlich verfügt die entsprechende Ausbildungsstätte nicht über das akademische Umfeld, das z. B. erlau- ben würde, große wissenschaftliche klinische Studien aufzulegen. Dennoch versetzt dieses intensive Pflegestudium die Studenten in die Lage, das Patientenmanagement besser zu verstehen, zu analysieren und zu helfen, praktische Konsequenzen daraus umzusetzen. Damit kann die Pflege in Zukunft auf gleicher Augenhöhe mit den Ärzten einen wesentlichen Beitrag zur Implementierung von evidenzbasierten Versorgungsleitlinien leisten.

\section{Korrespondenzadresse}

\section{Prof. Dr. W.A. Scherbaum}

Klinik für Endokrinologie,

Diabetologie und Rheumatologie,

European Training Centre in Endocrinology and Metabolism,

Universitätsklinikum Düsseldorf

Moorenstr. 5, 40225 Düsseldorf

scherbaum@uni-duesseldorf.de

Interessenkonflikt. Der korrespondierende Autor gibt für sich und seinen Koautor an, dass kein Interessenkonflikt besteht.

\section{Literatur}

1. Scherbaum WA, Landgraf R, Selbmann HK et al (2008) Diabetes-Leitlinien: Gemeinsames Handeln aller Beteiligten. Dtsch Artebl 105: A-1734-1736

2. Gary TL, Batts-Turner M, Yeh H-C et al (2009) The effects of a nurse case manager and a community health worker team on diabetic control, emergency department visits, and hospitalizations among urban African Americans with type 2 diabetes mellitus. Arch Intern Med 169:1788-1894

3. Welch G, Garb J, Zagarins S et al (2010) Nurse diabetes case management interventions and blood glucose control: results of a meta-analysis. Diabetes Res Clin Pract 88:1-6

4. Ishani A, Greer N, Taylor BC et al (2011) Effect of nurse case management compared with usual care on controlling cardiovascular risk in patients with diabetes: a randomized controlled trial. Diabetes Care 34:1689-1694

5. Appel SJ (2012) Nurse case management with a therapeutic algorithm for people living with diabetes, hypertension and raised LDL cholesterol: after 1 year $22 \%$ of those receiving the intervention have all three parameters under control versus $10 \%$ of those receiving usual care. Comment on Ishani A, Greer N, Taylor BC et al Diabetes Care 2011; 34:1689-1694. Evid Based Nurs 15:18-19

6. Risse A, Hochlehnert D (2010) Integrierte Versorgung. Diabetologe 6:100-107

7. Wernecke J, Friedl A (2010) Therapieziele beim geriatrischen Patienten. Diabetologe 6:551-559

8. Icks A, Chernayak N, Kleibn A et al (2011) Kosteneffektivität der Primärprävention des Typ-2-Diabetes. Diabetologe 7:92-98

\section{Kostenlose Broschüre: „Diabetes und Alzheimer: Risiken kennen und meiden"}

Gibt es einen Zusammenhang zwischen Diabetes und Alzheimer? Wie wirkt sich der Blutzuckerspiegel auf die geistige Leistungsfähigkeit aus? Die Alzheimer Forschung Initiative e.V. (AFI) beantwortet in der neuen Broschüre „Diabetes und Alzheimer: Risiken kennen und meiden" die wichtigsten Fragen zu diesem Thema. Zunächst wird ein Überblick zu Typ-2Diabetes und die Alzheimer-Krankheit gegeben. Anschließend wird das Zusammenspiel beider Erkrankungen beleuchtet. Im Schlusskapitel steht dann die Vermeidung von Risikofaktoren für Diabetespatienten im Mittelpunkt.

Die Broschüre „Diabetes und Alzheimer: Risiken kennen und meiden" kann kostenfrei bestellt werden, per Post bei der Alzheimer Forschung Initiative e.V.,

Kreuzstr. 34,

40210 Düsseldorf,

per Internet auf www.alzheimer-forschung. de/Rubrik Aufklärung, per E-Mail: info@alzheimer-forschung.de oder einfach über die gebührenfreie Telefonnummer 08002004001 .

Die Alzheimer Forschung Initiative (AFI) ist ein eingetragener gemeinnütziger Verein. Seit 1995 fördert die AFI mit Spendengeldern Forschungsprojekte engagierter Alzheimer-Forscher.

Quelle: Alzheimer Forschung Initiative (AFI), www.alzheimer-forschung.de 